\title{
A Metabonomics Profiling Study on Phlegm Syndrome and Blood-Stasis Syndrome in Coronary Heart Disease Patients Using Liquid Chromatography/Quadrupole Time-of-Flight Mass Spectrometry
}

\author{
Linlin Zhao, Ling Wan, Xinjian Qiu, Ruomeng Li, Shimi Liu, and Dongsheng Wang \\ Integrated Traditional Chinese and Western Medicine, Xiangya Hospital, Central south University, Changsha 410008, China \\ Correspondence should be addressed to Dongsheng Wang; wdsh666@126.com
}

Received 12 March 2014; Revised 20 May 2014; Accepted 30 May 2014; Published 20 July 2014

Academic Editor: Aiping Lu

Copyright (c) 2014 Linlin Zhao et al. This is an open access article distributed under the Creative Commons Attribution License, which permits unrestricted use, distribution, and reproduction in any medium, provided the original work is properly cited.

A metabonomics approach based on liquid chromatography/quadrupole time-of-flight mass spectrometry (LC-Q-TOF/MS) was utilized to obtain potential biomarkers of coronary heart disease (CHD) patients and investigate the ZHENG types differentiation in CHD patients. The plasma samples of $20 \mathrm{CHD}$ patients with phlegm syndrome, $20 \mathrm{CHD}$ patients with blood-stasis syndrome, and 16 healthy volunteers were collected in the study. 26 potential biomarkers were identified in the plasma of CHD patients and 19 differential metabolites contributed to the discrimination of phlegm syndrome and blood-stasis syndrome in CHD patients (VIP > $1.5 ; P<0.05)$ which mainly involved purine metabolism, pyrimidine metabolism, amino acid metabolism, steroid biosynthesis, and arachidonic acid metabolism. This study demonstrated that metabonomics approach based on LC-MS was useful for studying pathologic changes of CHD patients and interpreting the differentiation of ZHENG types (phlegm and blood-stasis syndrome) in traditional Chinese medicine (TCM).

\section{Introduction}

Coronary heart disease (CHD) usually associates with high morbidity and mortality; the incidence of CHD increases year by year and shows younger tendency [1]. Coronary angiography has been the "golden standard" for the diagnosis of CHD $[2,3]$. CHD is considered a "thoracic obstruction" (Xiongbi in Mandarin) in traditional Chinese medicine (TCM) $[3,4]$. Blood-stasis syndrome and phlegm syndrome are identified as the main ZHENG types (TCM syndrome or pattern) in patients with CHD [5]. ZHENG types are essential element of TCM theory and classified according to symptoms; different therapies result from different ZHENG types. The biological basis in microcosmic level of ZHENG types has been a study hot spot on standardization and modernization of TCM [6]. Metabonomics is defined as "the quantitative measurement of the dynamic multiparametric response of a living system to pathophysiological stimuli or genetic modification" $[7,8]$. It provide "an accurate, noninvasive and rapid diagnosis of coronary heart disease" [9]. Moreover its systemic, holistic, and dynamic characteristics are coincident with TCM theory, and it has been used to study ZHENG types differentiation and curative effects of Chinese medicine [10-13]. Analytical platforms of metabonomics include ${ }^{1} \mathrm{H}$ NMR, FT-IR, GC-MS, GC $\times$ GC-MS, LC-MS, and CE-MS [14-16]. Considering the complexity of the metabolites, none of the analytical platforms can be used alone to find all of the metabolites and a combination of methods is necessary to obtain as many metabolites as possible $[16,17]$. In our study, CHD patients with phlegm and blood-stasis syndrome were selected applying liquid chromatography quadrupole time-of-flight mass spectrometry (LC-QTOF/MS) to study the underlying essence of the disease (CHD) and ZHENG types (phlegm syndrome and blood-stasis syndrome).

\section{Method}

2.1. Subjects. $40 \mathrm{CHD}$ patients (20 with phlegm syndrome and 20 with blood-stasis syndrome), confirmed by coronary angiography, came from Xiangya Hospital affiliated to 
Central South University (Hunan, China) from March 1, 2012, to June 30, 2013. Diagnosis standard of CHD based on "nomenclature and diagnosis criteria of ischemic heart disease" is established by the Joint International Society and Federation of Cardiology/World Health Organization Task Force on Standardization of Clinical Nomenclature [18]. The ZHENG types were identified by three chief physicians, according to "criteria for TCM syndrome differentiation of patients with coronary heart disease" [19].

16 age and sex matched healthy volunteers were enrolled from medical examination center in the same hospital. A questionnaire was used to survey lifestyle and drugs intake within a week. The clinical data of each subject were shown in the Supplementary Material available online at http://dx.doi.org/10.1155/2014/385102.

Excluded cases contained patients who suffered from diabetic cardiomyopathy, hyperthyroid heart disease, hypertensive heart disease, pulmonary heart disease, anemic heart disease, systemic scleroderma heart disease, inborn coronary abnormity, and rheumatic heart disease, who suffered from severe hypertension, malignant tumor, renal failure, thyroid disease, diabetes mellitus, and pulmonary infection, who suffered from infectious diseases, such as hepatitis and tuberculosis, and who suffered from invigorative system disease and women in pregnant or in lactation.

The study was approved by the hospital ethics committee and all subjects gave written informed consent.

2.2. Chemicals. HPLC-grade acetonitrile, methanol, formic acid, and ammonium formate were obtained from Merck \& Co Inc. (NJ, USA). Water was distilled thrice from silica glass equipment before use.

2.3. Samples Collection and Preparation. $5 \mathrm{~mL}$ venous plasma samples were collected into ethylenediaminetetraacetic acid (EDTA) vacutainer tubes in the morning after $12 \mathrm{~h}$ of overnight fasting and then centrifuged at $10000 \mathrm{rpm}$ for $10 \mathrm{~min}$ at $4^{\circ} \mathrm{C}$; the supernatant was frozen at $-80^{\circ} \mathrm{C}$ until analysis.

The samples at room temperature thawed for $15 \mathrm{~min}$ and vortexed for $5 \mathrm{~s}$ before use. Each $100 \mu \mathrm{L}$ of plasma was diluted with $300 \mu \mathrm{L}$ methanol; after being vortexed for $30 \mathrm{~s}$, the mixture was stored at $4^{\circ} \mathrm{C}$ for $10 \mathrm{~min}$ and then centrifuged at $12000 \mathrm{rpm}$ and $4^{\circ} \mathrm{C}$ for $15 \mathrm{~min}$. The supernatant was extracted to autosampler.

2.4. LC-MS Instrumentation and Analytical Condition. The assay was performed on LC-Q/TOF-MS using Agilent 1290 Infinity LC coupled with an electrospray ionization (ESI) source and Agilent 6530Q-TOF mass spectrometer (Agilent Technologies, Palo Alto, CA, USA). The LC separation was carried out on a ZORBAX RRHD SB-C ${ }_{18}$ column (2.1 $\times 100 \mathrm{~mm}, 1.8 \mu \mathrm{m}$, Agilent) with the column temperature maintained at $40^{\circ} \mathrm{C}$. The mobile phases consisted of ultrapure water (A) and acetonitrile (B) both containing $0.1 \%(\mathrm{v} / \mathrm{v})$ formic acid; the gradient program was shown in Table 1. The injected volume of sample was $4 \mu \mathrm{L}$ and temperature of autosampler was $4^{\circ} \mathrm{C}$.
TABLE 1: The gradient program of mobile phase.

\begin{tabular}{lccc}
\hline Time $(\mathrm{min})$ & Flow rate $(\mathrm{mL} / \mathrm{min})$ & $\mathrm{A}(\%)$ & $\mathrm{B}(\%)$ \\
\hline 0 & 0.4 & 95 & 5 \\
2 & 0.4 & 95 & 5 \\
17 & 0.4 & 5 & 95 \\
19 & 0.4 & 5 & 95 \\
\hline
\end{tabular}

Nitrogen was used as both nebuliser gas and cone gas. Detection mode of flight tube was type V. The mass spectrometric data was collected in both positive and negative modes with the following parameters: capillary voltage $4 \mathrm{kV}$ (positive mode) and $3.5 \mathrm{kV}$ (negative mode), sampling cone voltage $35 \mathrm{kV}$ (positive mode) and $50 \mathrm{kV}$ (negative mode), source temperature $100^{\circ} \mathrm{C}$, desolvation temperature $350^{\circ} \mathrm{C}$ (positive mode) and $300^{\circ} \mathrm{C}$ (negative mode), cone gas flow rate $50 \mathrm{~L} / \mathrm{h}$, desolvation gas flow rate $600 \mathrm{~L} / \mathrm{h}$ (positive mode) and $700 \mathrm{~L} / \mathrm{h}$ (negative mode), extraction cone voltage $4 \mathrm{~V}$, and full scan mode scanning from $m / z 50-1000$ with a scan time of $0.03 \mathrm{~s}$ and an interscan time of $0.02 \mathrm{~s}$. Leucine enkephalin was used as the lock mass $\left([\mathrm{M}+\mathrm{H}]^{+}=556.2771\right.$ in the positive mode and $[\mathrm{M}-\mathrm{H}]^{-}=554.2615$ in the negative mode).

2.5. Data Processing and Analysis. All mass spectral data were collected in .d format and converted to .mz Data using the Mass Hunter Qualitative Analysis software (Version B 03.01, Agilent Technologies, Palo Alto, CA, USA); XCMS program of R software platform was used for data preprocessing, such as baseline filtering, peak identification, nonlinear retention time alignment, and peak matching. The next data were performed in Excel 2007, and the data were combined to form the final two-dimensional matrix. 958 metabolites in positive mode and 1589 metabolites in negative mode were detected.

To determine distinction between groups, multivariate analyses including principal components analysis (PCA) and partial least squares-discriminate analysis (PLS-DA) $[20,21]$ were conducted with Simca-P software 11.0 (Umetrics, Umea, Sweden). To obtain more reliable and intuitive results, unit variance (UV) scaling and mean-centered were applied to analyzing the data.

The $R^{2} Y$ and $Q^{2}$ values were used to evaluate the model quality, which indicate the fit $\left(R^{2} Y\right)$ and prediction ability $\left(Q^{2}\right) ; R^{2} Y$ and $Q^{2}$ values closing to 1.0 means an excellent mathematical model and the values $>0.5$ suggest reliable predictive accuracy. Variable importance projection (VIP) constructed from PLS-DA analysis was used to preselect variables (VIP $>1.0$ means influential and VIP $\geq 1.5$ means highly influential) and student's $t$ test $(P<0.05)$ was selected to measure the significance of each differential metabolite. The metabolites were qualified by the Scripps Center for Metabonomics and Mass Spectrometry (METLIN, http://metlin.Scripps.edu/) and the Human Metabolome Database (HMDB, http://hmdb.ca); the possible pathways of metabolites were obtained from the Kyoto Encyclopedia of Genes and Genomes (KEGG, http://www.genome.jp/kegg/) and HMDB. 


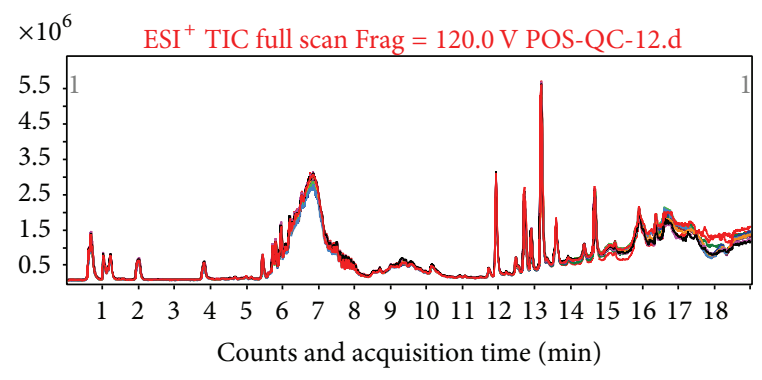

(a)

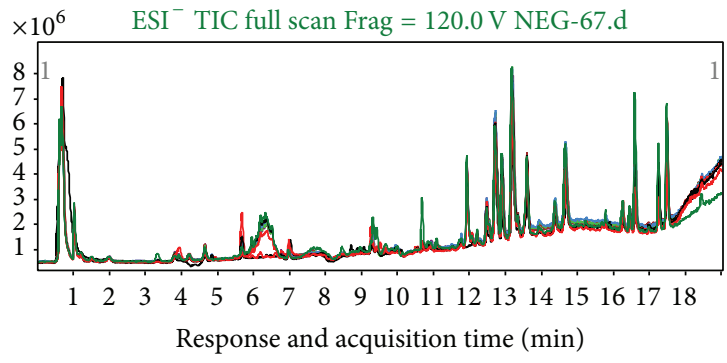

(b)

FIGURE 1: Overlapping total ion current (TIC) chromatograms of plasma samples in (a) positive mode and (b) negative mode.

TABLE 2: Demographic and clinical characteristics of subjects.

\begin{tabular}{|c|c|c|c|}
\hline Characteristics & $\begin{array}{l}\text { Blood stasis syndrome } \\
\qquad n=20\end{array}$ & $\begin{array}{c}\text { Phlegm syndrome } \\
n=20\end{array}$ & $\begin{array}{c}\text { Healthy } \\
n=16\end{array}$ \\
\hline Age (years) & $61.05 \pm 11.77$ & $62.08 \pm 10.15$ & $60.27 \pm 12.04$ \\
\hline Male & $13(65 \%)$ & $17(85 \%)$ & $10(62.5 \%)$ \\
\hline BMI $\left(\mathrm{kg} / \mathrm{m}^{2}\right)$ & $23.11 \pm 1.45$ & $27.72 \pm 0.86$ & $23.60 \pm 0.87$ \\
\hline Hypertension & $6(30 \%)$ & $10(50 \%)$ & $6(37.5 \%)$ \\
\hline $\mathrm{TG}(\mathrm{mmol} / \mathrm{L})$ & $1.63 \pm 0.90$ & $1.54 \pm 1.02$ & $1.23 \pm 0.77$ \\
\hline $\mathrm{TC}(\mathrm{mmol} / \mathrm{L})$ & $4.15 \pm 0.98$ & $4.11 \pm 0.93$ & $3.25 \pm 0.72$ \\
\hline $\mathrm{LDL}(\mathrm{mmol} / \mathrm{L})$ & $2.21 \pm 0.93$ & $2.08 \pm 0.93$ & $2.02 \pm 0.44$ \\
\hline $\mathrm{HDL}(\mathrm{mmol} / \mathrm{L})$ & $1.19 \pm 0.24$ & $1.14 \pm 0.48$ & $1.27 \pm 0.28$ \\
\hline $\operatorname{Apo}\left(a_{1}\right)(g / L)$ & $1.29 \pm 0.21$ & $1.17 \pm 0.21$ & $1.33 \pm 0.14$ \\
\hline Apo (b) (g/L) & $1.04 \pm 0.33$ & $0.77 \pm 0.19$ & $0.62 \pm 0.30$ \\
\hline LDH (U/L) & $225.78 \pm 137.61$ & $206.31 \pm 56.52$ & $158.01 \pm 42.21$ \\
\hline $\mathrm{CK}(\mathrm{U} / \mathrm{L})$ & $253.96 \pm 691.83$ & $81.39 \pm 45.08$ & $73.30 \pm 11.68$ \\
\hline CK-MB (U/L) & $19.27 \pm 21.95$ & $19.11 \pm 10.02$ & $24.45 \pm 14.80$ \\
\hline Myoglobin (ug/L) & $41.80 \pm 29.09$ & $43.28 \pm 22.07$ & $32.40 \pm 10.67$ \\
\hline CTn1 (ug/L) & $0.12 \pm 0.21$ & $0.44 \pm 0.29$ & $0.17 \pm 0.11$ \\
\hline
\end{tabular}

\section{Results}

3.1. Demographic and Clinical Characteristics. Demographic and clinical data of subjects were summarized in Table 2.

3.2. Visual Inspection of Chromatography. In order to inspect the performance of LC-MS instrumentation, three blood samples were selected randomly in the positive and negative mode, respectively, for visual inspection; overlapping total ion current (TIC) chromatograms were shown in Figure 1. There appeared to be strong instrumental analysis signal, highlighting peaks capacity and good retention time reproducibility.

3.3. Metabolic Profiles of CHD Patients Compared to Healthy Subjects and Detection of Potential Metabolic Biomarkers. The PCA scores plots showed significant discrimination between the CHD group and the healthy control group in both positive (Figure 2(a)) and negative modes (Figure 2(b)). In the score plot, the confidence interval was defined by the Hotelling $T^{2}$ ellipse (95\% confidence interval). As exhibited in Figure 2, most samples were within the $95 \%$ confidence interval.
PLS-DA models confirmed the clear separation tendency between the two groups. The classification results were shown in Figures 2(c) and 2(d). All the models parameters were presented in Table 3.

VIP (from PLS-DA) $>1.5$ and $P$ value (from student's $t$ test $)<0.05$ were used to confirm the differential metabolites. Retention time (RT), mass-to-charge ratio $(\mathrm{m} / \mathrm{z})$, differential metabolites, VIP value, $P$ value, and fold (changed trend of $\mathrm{CHD}$ patients compared to the healthy group) were presented in Table 4 simultaneously. The concentrations of 3 metabolites were significantly upregulated (compared to the healthy controls) in the CHD patients, while the remaining 23 metabolites were significantly downregulated.

3.4. ZHENG Differentiation. The corresponding models scores plots of the ZHENG types and the parameters were exhibited in Figure 3 and Table 2, respectively. As shown in Figure 3 most samples were within the scope Hotelling $T^{2}$ ellipse. PLS-DA models in both $\mathrm{ESI}^{+}$(Figure 3(c)) and $\mathrm{ESI}^{-}$ (Figure 3(d)) modes and PCA model in $\mathrm{ESI}^{-}$(Figure 3(b)) mode illustrated that there was satisfactory classification between phlegm syndrome and blood-stasis syndrome, 
TABLE 3: Summary of the parameters for modeling quality.

\begin{tabular}{lccccccr}
\hline \multirow{2}{*}{ Group } & \multirow{2}{*}{ Mode } & \multicolumn{3}{c}{ PCA model } & \multicolumn{3}{c}{ PLS-DA model } \\
& & ${ }^{\mathrm{e}} \mathrm{No}$ & $R^{2} X$ & $Q^{2} Y$ & ${ }^{\mathrm{e}} \mathrm{No}$ & $R^{2} X$ & $R^{2} Y$ \\
\hline CHD/healthy & $\mathrm{ESI}^{+}$ & 2 & 0.433 & 0.378 & 2 & 0.376 & 0.993 \\
CHD/healthy & $\mathrm{ESI}^{-}$ & 3 & 0.349 & 0.187 & 3 & 0.237 & 0.989 \\
A/B & $\mathrm{ESI}^{+}$ & 2 & 0.430 & 0.349 & 4 & 0.508 & 0.989 \\
A/B & $\mathrm{ESI}^{-}$ & 3 & 0.279 & 0.105 & 3 & 0.253 & 0.969 \\
\hline
\end{tabular}

A represented phlegm syndrome in CHD, B represented blood-stasis syndrome in CHD, and ${ }^{\mathrm{e}}$ No represented amount of components.

TABLE 4: Plasma metabolites for discriminating CHD patients from healthy controls.

\begin{tabular}{|c|c|c|c|c|c|c|}
\hline Mode & RT & Mass & Metabolite & VIP & $P$ & ${ }^{\mathrm{a}}$ Fold \\
\hline \multirow{15}{*}{ EIS $^{+}$} & 6.21 & 542.28 & Cortolone-3-glucuronide & 1.75 & $7.02 E-44$ & -25.59 \\
\hline & 5.74 & 431.21 & 17-Phenoxy trinor PGF2 $\alpha$ ethyl amide & 1.74 & $1.59 E-37$ & -25.32 \\
\hline & 5.71 & 562.37 & Cholesterol glucuronide & 1.74 & $1.34 E-35$ & -25.35 \\
\hline & 5.03 & 362.22 & Cortisol & 1.73 & $7.10 E-34$ & -24.65 \\
\hline & 7.52 & 890.51 & Dipalmitoyl phosphatidylinositol 3-phosphate & 1.73 & $1.28 E-33$ & -25.53 \\
\hline & 15.91 & 370.24 & TXB2 & 1.73 & $3.64 E-31$ & -24.54 \\
\hline & 13.41 & 393.3 & PGH2-EA & 1.71 & $2.30 E-28$ & -25.08 \\
\hline & 15.90 & 298.18 & 13,14-Dihydro-15-keto-tetranor PGE2 & 1.70 & $5.56 E-27$ & -25.84 \\
\hline & 15.90 & 372.25 & TXB1 & 1.70 & $1.42 E-26$ & -26.76 \\
\hline & 11.98 & 338.17 & 18-Carboxy dinor leukotriene B4 & 1.70 & $1.55 E-26$ & -24.83 \\
\hline & 8.98 & 413.26 & 15-Keto-17-phenyl trinor Prostaglandin F2 $\alpha$ ethyl amide & 1.66 & $8.12 E-22$ & -24.57 \\
\hline & 14.52 & 510.28 & Leukotriene D4 methyl ester & 1.65 & $8.28 E-21$ & -22.56 \\
\hline & 6.71 & 427.27 & 17-Phenyl trinor prostaglandin F2 $\alpha$ Cyclopropyl amide & 1.61 & $1.41 E-18$ & -24.27 \\
\hline & 16.05 & 348.23 & PGA2 methyl ester & 1.59 & $2.34 E-17$ & -22.23 \\
\hline & 5.77 & 472.24 & Chenodeoxycholic acid 3-sulfate & 1.50 & $1.24 E-13$ & -23.70 \\
\hline \multirow{12}{*}{ EIS $^{-}$} & 1.03 & 244.07 & Uridine & 1.98 & $7.81 E-12$ & -0.72 \\
\hline & 16.61 & 348.23 & PGA2 methyl ester & 1.98 & $9.09 E-12$ & 3.52 \\
\hline & 1.02 & 136.04 & Hypoxanthine & 1.90 & $1.66 E-10$ & -1.84 \\
\hline & 1.03 & 192.03 & Citric acid & 1.89 & $2.40 E-10$ & 2.12 \\
\hline & 3.81 & 204.09 & L-Tryptophan & 1.88 & $3.85 E-10$ & -1.01 \\
\hline & 3.81 & 324.03 & Uridine monophosphate (UMP) & 1.81 & $3.09 E-09$ & -1.98 \\
\hline & 2.00 & 165.08 & L-Phenylalanine & 1.61 & $5.73 E-07$ & -1.30 \\
\hline & 4.66 & 282.11 & 2-Aminoadenosine & 1.56 & $1.57 E-06$ & -2.41 \\
\hline & 13.16 & 625.30 & Leukotriene C4 & 1.55 & $1.83 E-06$ & -2.20 \\
\hline & 16.62 & 302.22 & EPA & 1.55 & $1.87 E-06$ & -2.47 \\
\hline & 16.88 & 336.23 & LTB4 & 1.53 & $2.68 E-06$ & 4.50 \\
\hline & 1.91 & 116.05 & 2-Keto valeric acid & 1.51 & $4.25 E-06$ & -2.05 \\
\hline
\end{tabular}

${ }^{\mathrm{a}}$ Fold $=\log _{2}$ (average peak intensity of CHD group/average peak intensity of healthy group), “-” represented downregulated compared to healthy group, and "+" represented upregulated compared to healthy group.

which reflected the pathological variation between the two ZHENG types.

The differential metabolites contributing to the clustering and discrimination (VIP $>1.5 ; P<0.05$ ) between phlegm syndrome and blood-stasis syndrome were presented in Table 5; there were 7 metabolites' concentrations in phlegm syndrome significantly higher than that in blood-stasis syndrome while the remaining 11 metabolites were contrary.

\section{Discussion}

Metabonomics profiling study on CHD patients has been investigated many times. For instance, Brindle et al. have reported for the first time that metabonomics analysis of serum samples based on ${ }^{1} \mathrm{H}-\mathrm{NMR}$ can provide a clinically useful diagnosis of $\mathrm{CHD}$ and can be used as a replacement of angiography. Metabolic profiling of biological specimens could be of great significance for clinicians and biologists in the early diagnosis [9]. Sun et al. find 16 differential metabolites between unstable angina (UA) and atherosclerosis patients, which are closely associated with phospholipid metabolism, tryptophan metabolism, and MG metabolism. These potential biomarkers can provide information on understanding the biological mechanisms of UA and the diagnosis of UA [22]. In our previous studies with GC-MS technology, we obtained 32 potential biomarkers of $\mathrm{CHD}$ 
TABle 5: Plasma differential metabolites for discriminating phlegm from blood-stasis syndrome.

\begin{tabular}{|c|c|c|c|c|c|c|}
\hline Mode & $\mathrm{RT}$ & Mass & Metabolite & VIP & $P$ & ${ }^{\mathrm{a}}$ Fold \\
\hline \multirow{11}{*}{$\mathrm{EIS}^{+}$} & 1.21 & 131.10 & L-Leucine & 1.83 & $9.54 E-04$ & -0.62 \\
\hline & 1.02 & 168.03 & Uric acid & 1.73 & $1.91 E-03$ & -1.32 \\
\hline & 2.00 & 165.08 & L-Phenylalanine & 1.68 & $2.81 E-03$ & -0.37 \\
\hline & 3.82 & 204.09 & L-Tryptophan & 1.62 & $4.05 E-03$ & -0.26 \\
\hline & 10.94 & 250.12 & Ubiquinone & 1.57 & $5.38 E-03$ & -0.81 \\
\hline & 15.21 & 384.29 & 17,20-dimethyl prostaglandin F1 $\alpha$ & 1.57 & $5.61 E-03$ & 0.75 \\
\hline & 3.82 & 145.05 & Isoquinoline $\mathrm{N}$-oxide & 1.55 & $6.08 E-03$ & -0.26 \\
\hline & 10.96 & 393.29 & $\mathrm{PGH}_{2}$-EA & 1.54 & $6.46 E-03$ & 0.43 \\
\hline & 17.48 & 283.29 & Stearamide & 1.54 & $6.56 E-03$ & -1.71 \\
\hline & 11.74 & 568.33 & Deoxycholic acid 3-glucuronide & 1.52 & $7.62 E-03$ & 0.88 \\
\hline & 16.85 & 368.29 & Octadecyl fumarate & 1.50 & $8.10 E-03$ & 1.10 \\
\hline \multirow{9}{*}{ EIS $^{-}$} & 10.28 & 250.12 & Ubiquinone & 2.70 & $5.97 E-10$ & -1.15 \\
\hline & 17.23 & 363.24 & N-Palmitoyl taurine & 2.58 & $1.05 E-08$ & -2.33 \\
\hline & 17.46 & 389.26 & $\mathrm{~N}$-Oleoyl taurine & 2.57 & $1.11 E-08$ & -1.95 \\
\hline & 16.68 & 552.19 & Deoxycholic acid disulfate & 2.26 & $2.43 E-06$ & -2.08 \\
\hline & 1.08 & 181.07 & L-Tyrosine & 1.76 & $5.73 E-04$ & 0.56 \\
\hline & 1.03 & 244.07 & Uridine & 1.70 & $9.09 E-04$ & -0.28 \\
\hline & 0.74 & 130.03 & Itaconic acid & 1.68 & $1.06 E-03$ & 1.44 \\
\hline & 1.04 & 308.04 & Deoxyuridine monophosphate (dUMP) & 1.63 & $1.64 E-03$ & -1.04 \\
\hline & 16.75 & 330.26 & Eicosapentaenoic acid ethyl ester & 1.63 & $1.65 E-03$ & 4.77 \\
\hline
\end{tabular}

${ }^{a}$ Fold $=\log _{2}$ (average peak intensity of phlegm syndrome/average peak intensity of blood stasis syndrome); "-" represented downregulated compared to blood stasis syndrome; "+" represented upregulated compared to blood stasis syndrome.

patients, including amino acids, fatty acids, organic acids, glucose, and alcohol esters [23].

In the research, 26 potential biomarkers that discriminated CHD patients from healthy controls were obtained. We found that the potential biomarkers were principally involved in arachidonic acid metabolism, amino acid metabolism, purine metabolism, pyrimidine metabolism, steroid biosynthesis, and linoleic acid metabolism, which were mainly correlated to inflammation, amino acid dysfunction, energy metabolism dysfunction, and oxidative injury in the pathological development of CHD. The disturbed metabolic pathway was shown in Figure 4.

(1) Inflammation: four biomarkers including TXB1, TXB2, leukotriene C4, and Leukotriene B4 belong to the pathway of arachidonic acid metabolism which could cause inflammation and lipid infiltration [24]. The leukotrienes have been identified as primarily proinflammatory mediators. LTB4 can activate leukocytes and promote conversion of monocyte into foam cells. Increased leukocytes adsorb to vascular endothelium [25]; foam cells accumulate and form fatty streak and lipid plague. LTC4 increase permeability of vascular in postcapillary venules and lead to potent vasoconstrictive effects on coronary arteries [26]. The result supported that activation of inflammation plays a role in the pathogenesis of CHD.

(2) Amino acid dysfunction: concentrations of tryptophan and phenylalanine were lower in the plasma of the CHD group than the healthy group which indicated that the amino acid metabolic pathways were disrupted in CHD patients. Tryptophan is considered an important mechanism in immune responses. Tryptophan catabolism suppresses helper $\mathrm{T}$ cell (Thl) activity [27]; the latter can activate macrophages, contribute to the activation of inflammation like delayed hypersensitivity $[28,29]$, and promote the formation of coronary atherosclerotic plaque. Consequently, degradation of tryptophan reflects an anti-inflammatory pathway and counterregulatory protective mechanism on early atherosclerotic lesions. However, permanent state of immune activation and low level of tryptophan may contribute to development of immunodeficiency [30]. Downregulation of tryptophan has been demonstrated in other studies about CHD [31, 32], and similar relationships between diminished tryptophan and immune activation are found in gynecological cancer, sepsis [33], rheumatoid arthritis [34], and Parkinson's disease [35]. Another pathway of tryptophan is the generation of the neurotransmitter 5-hydroxytryptamine (5-HT), which is a strong modulator of emotional behavior in the central nervous system. Decreased tryptophan induces declined 5HT which was possibly responsible for depressive mode and sleep disorder of CHD patients.

Phenylalanine is metabolised to tyrosine, and the latter is decarboxylated to tyramine and also to Dopa, dopamine, and noradrenaline, as shown in Figure 4. Lower level of phenylalanine was consistent with previous study $[36,37]$ which is possibly related to neuropsychiatric disturbances [38].

(3) Energy metabolism dysfunction: there were two pathways including purine metabolism (hypoxanthine, 2aminoadenosine) and pyrimidine metabolism (uridine, uridine monophosphate) that contributed to energy imbalance 


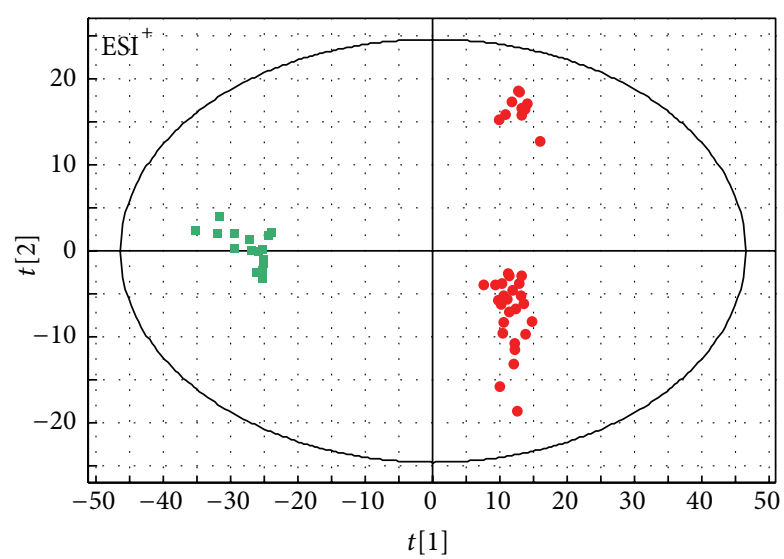

- $C$

- $A+B$

(a)

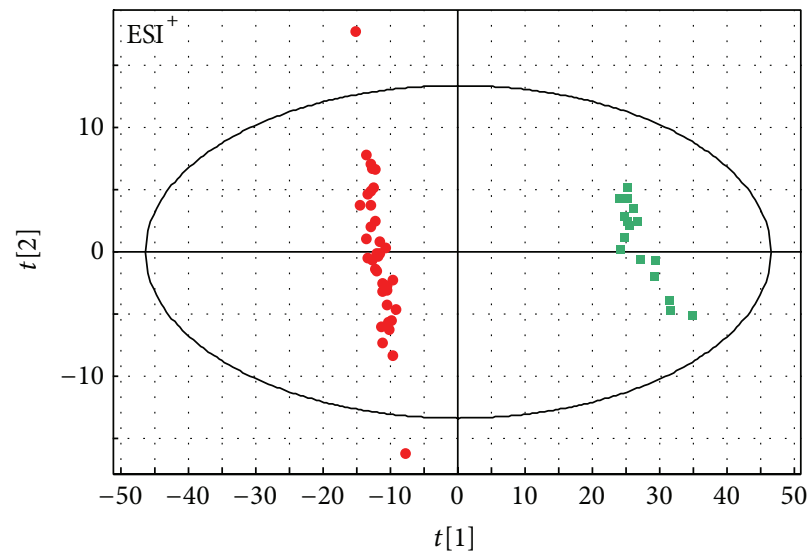

(c)

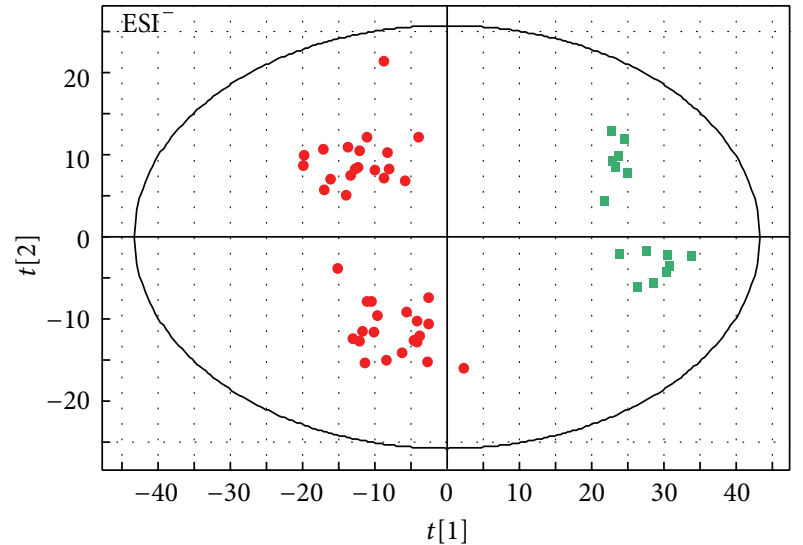

- $C$

- $A+B$

(b)

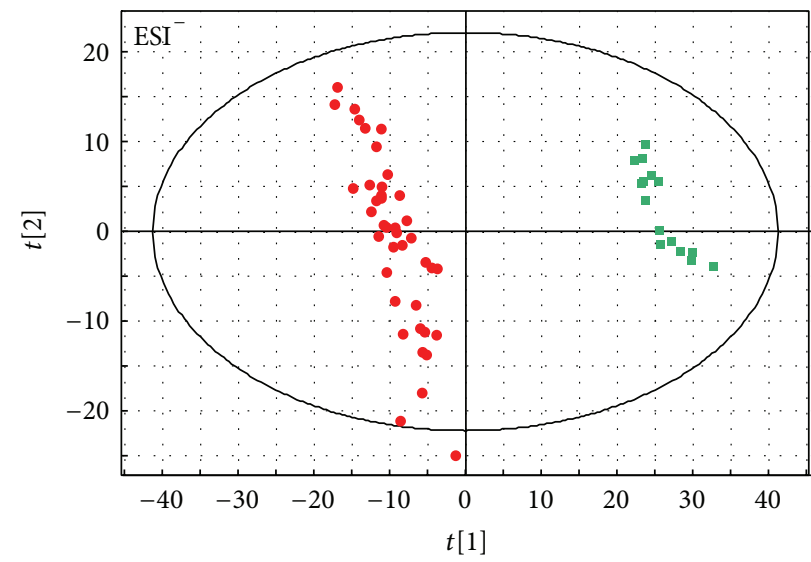

(d)

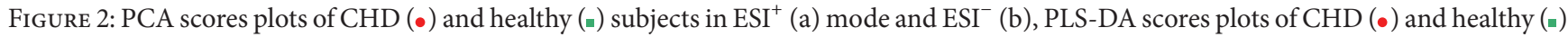
subjects in $\mathrm{ESI}^{+}$(c) mode and $\mathrm{ESI}^{-}$(d). $X$-axis expressed the first component of the metabolite data. $Y$-axis expressed the second component.

in CHD patients. When myocardial energy metabolism dysfunction occurs owing to ischemia, the nucleotide degradation pathway is activated. In fact, the relevance between myocardial ATP exhaustion and the increased hypoxanthine and uridine has been clearly observed [39]; in addition, uridine has been considered a marker of myocardial viability after coronary occlusion and reperfusion and an indirect indicator of tissue energy crisis [40]. Increased uridine and decreased hypoxanthine were found in previous metabonomics study of myocardial infarction in rats [31, 41]; however, both hypoxanthine and uridine downregulated in our study and drug medication possibly play a role. On the other hand, when hypoxanthine converts to xanthine, reactive oxygen species (ROS) generate which is the characteristic of hypoxia and play a major role in microvascular dysfunction and exert direct tissue damage [42]. Citric acid cycle obstacle result in the accumulation of citric acid in the plasma, oxalosuccinic acid belongs to the citric acid cycle, and increased oxalosuccinic acid is found in a study [31] which is kept in step with increased citric acid in our study. Multiple metabolites in a particular pathway correlating with the perturbation will be found in a further study which help us understand the mechanisms of CHD better.

(4) Other metabolic abnormalities: the concentrations of cortolone-3-glucuronide and cortisol involved in steroid metabolism and cholesterol glucuronide involved in cholesterol were lower in CHD patients in our study.

We noticed that there is no significant difference in lipid metabolites between CHD patients and healthy people which would be interpreted with the intervention of lipid-lowering medication. Drugs could confound the metabonomics data; however, we still found clear separate trend between the groups. We will select more cases to validate the result in a further study.

As shown in Figure 4, there were 18 differential metabolites mainly involved in amino acid metabolism (leucine, phenylalanine, tryptophan, and tyrosine), purine metabolism (uric acid, ubiquinone), and pyrimidine metabolism (dUMP, uridine), which exhibited a satisfactory classification between phlegm syndrome and blood-stasis syndrome in CHD 


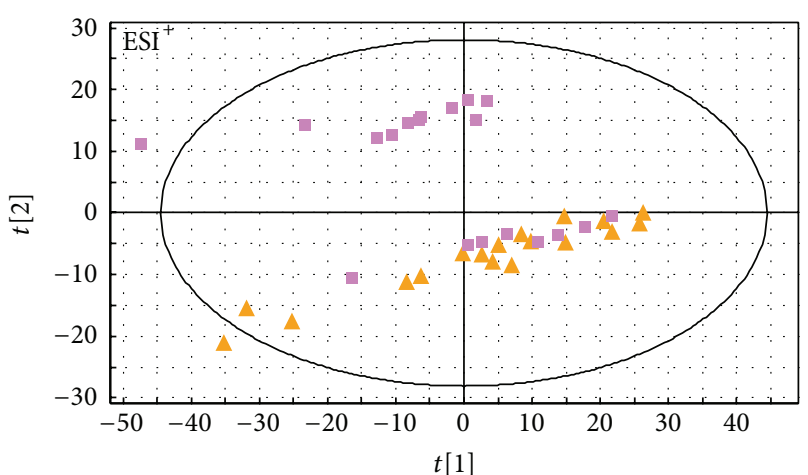

(a)

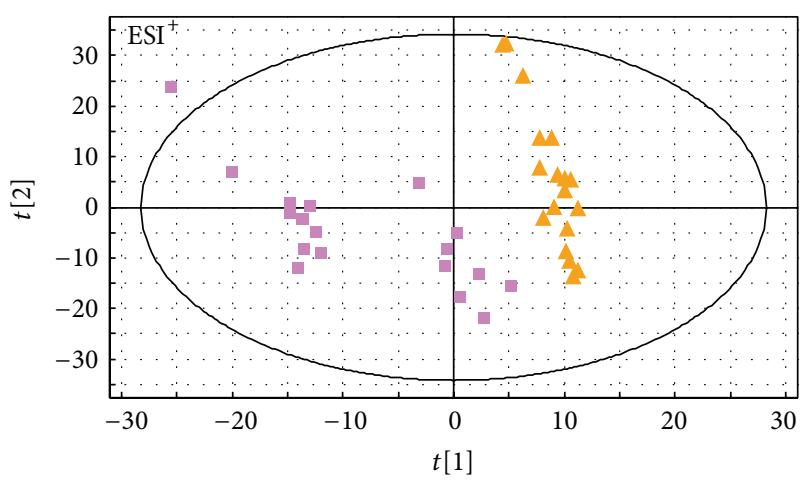

(c)

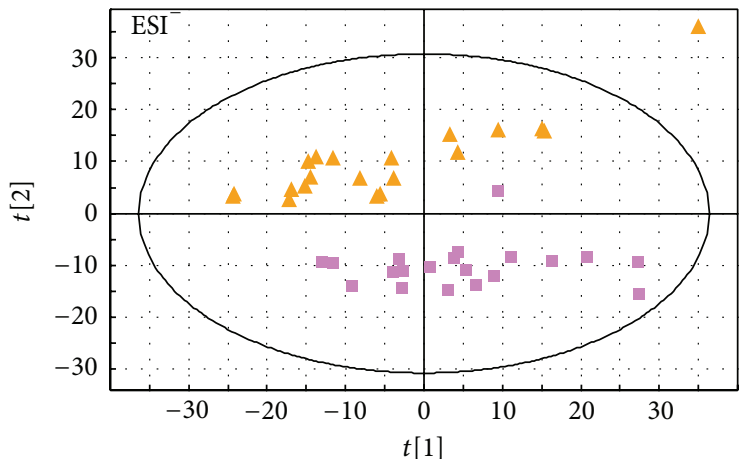

(b)

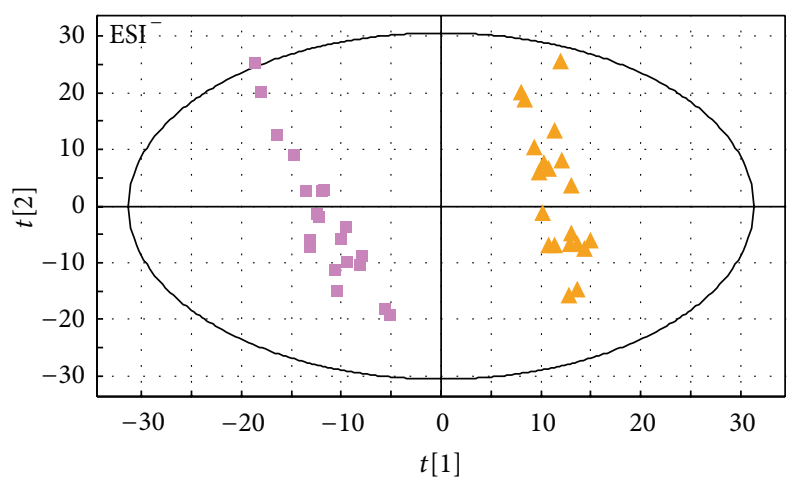

(d)

FIGURE 3: PCA scores plots of CHD with phlegm syndrome ( $₫$ ) and CHD with blood-stasis syndrome ( $\triangle$ ) in $\mathrm{ESI}^{+}$(a) mode and ESI $(\mathrm{b})$, PLS-DA scores plots of the two ZHENG types in $\mathrm{ESI}^{+}$(c) mode and $\mathrm{ESI}^{-}$(d). $X$-axis expressed the first component of the metabolite data. $Y$-axis expressed the second component.

patients. The levels of amino acid (except phenylalanine) were lower in phlegm syndrome than in blood-stasis syndrome of $\mathrm{CHD}$, which indicated different degrees of disorder in amino acid metabolism of the two ZHENG types. However, some study found that severity of the amino acid metabolic disorders in phlegm syndrome is similar to blood-stasis syndrome of CHD [43], the difference being possibly caused by using different metabonomics technology and analysis method. Increased phenylalanine to tyrosine ratio (Phe/Tyr) represents immune activation and higher Phe/Tyr was found in blood-stasis syndrome of CHD group.

Uridine, uric acid, and dUMP were increased in CHD patients with blood stasis compared to phlegm syndrome; uridine and uric acid were the main nucleotide catabolite of myocardial ischemia and the level of uric acid in plasma is significantly relevant to risk of cardiovascular atherosclerotic disease and cardiovascular disease mortality [38, 44-47]. Uric acid also contributed to oxidative injury [48]. dUMP was involved in oxidative injury and uridine related to energy metabolism.

The differences in inflammation, oxidative injury, and energy metabolism reflected phlegm syndrome and bloodstasis syndrome in CHD belonging to different metabolism patterns; in addition, they were possibly different stages of the CHD pathogenesis development [49]. Biomarkers confirmed in the further study will enhance the ability to identify
ZHENG types of phlegm and blood stasis in CHD and reduce the artificial error in identifying syndrome differentiation. It could provide widespread population screening and ZHENG types standardization in TCM.

As a preliminary study, we considered that LC-MS-based metabonomics can be useful to study the underlying essence of the disease (CHD) and ZHENG types (phlegm and blood syndrome). However, the sample size was small and the casecontrol design limits causality in this study; more subjects will be needed to confirm the potential biomarkers in subsequent research; meanwhile, we will use another technique, $\mathrm{H}^{1}$ $\mathrm{NMR}$, to validate the separated trend and acquire more potential biomarkers. In addition, the extraneous variables, such as age, gender, smoking habits, complications (such as hypertension, diabetes), and treatment will be stratified to improve reliability of these potential biomarkers. Furthermore, validation with other study populations will be needed to identify reliability of the models in the future study.

\section{Conclusions}

In our study, $\mathrm{CHD}$ subjects were distinguished from the healthy volunteers utilizing the method of metabonomics based on LC-Q-TOF/MS and multivariate statistical analysis (PCA and PLS-DA). Differential metabolites between $\mathrm{CHD}$ patients and healthy subjects were mainly involved 


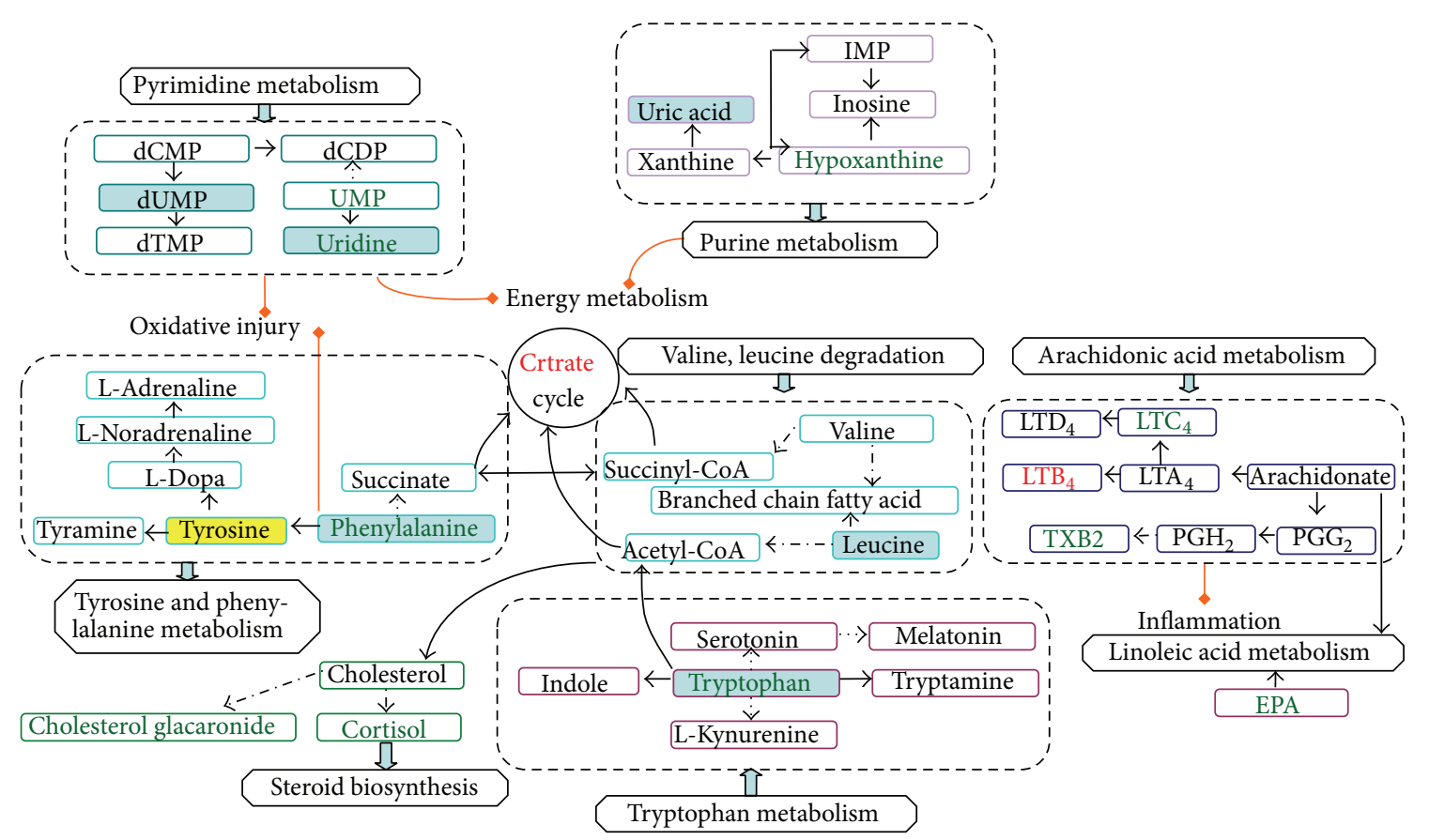

FIGURE 4: Disturbed metabolic pathways. Green font denoted biomarkers downregulations and red font denoted biomarkers upregulations (CHD/healthy people); yellow area denoted metabolites higher level and blue area denoted lower level (phlegm/blood stasis in CHD).

in arachidonic acid metabolism, amino acid metabolism, purine metabolism, pyrimidine metabolism, and steroid biosynthesis, which were related to inflammation, amino acid dysfunction, energy metabolism dysfunction, and oxidative injury. The two ZHENG types (phlegm and blood stasis) in this study were also obviously discriminated by metabonomics approach. The significant difference in concentration of metabolites demonstrated that they were probably different stages of the CHD pathogenesis development.

\section{Conflict of Interests}

The authors declare that there is no conflict of interests regarding the publication of this paper.

\section{Acknowledgments}

The work was supported by National Natural Science Foundation of China (81173198, 81373554), Scientific Foundation of Hunan China (09JJ5055), International cooperation Program of the S \& T Ministry, China (2010DFA32370), and Program of Hunan Provincial Science and Technology Department (2008WK3002, 2009JT4012).

\section{References}

[1] D. F. Jiang and J. C. Zhou, "Age characteristics analysis of 6295 patients with coronary heart disease," Medical Information Operations Sciences Fascicule, vol. 25, no. 4, pp. 1389-1390, 2011.

[2] L. Shi, C. Y. Yang, J. Y. Zhang, and H. Li, "Recognition of ST segment of electrocardiogram based on wavelet transform," Life Science Journal, vol. 4, no. 2, pp. 90-93, 2007.
[3] Q. Shi, H. H. Zhao, J. X. Chen et al., "Study on TCM syndrome identification modes of coronary heart disease based on data mining," Evidence-Based Complementary and Alternative Medicine, vol. 2012, Article ID 697028, 11 pages, 2012.

[4] J. Wang, P. Q. Wang, and X. J. Xiong, "Current situation and reunderstanding of syndrome and formula syndrome in Chinese medicine," Internal Medicine, vol. 2, article 113, 2012.

[5] J. X. Zhou, M. Tang, and J. Li, "Analysis of Chinese syndrome features and combination laws of 2029 patients with coronary heart disease angina," Chinese journal of integrated traditional and Western medicine, vol. 31, no. 6, pp. 753-755, 2011.

[6] C. Matsumoto, T. Kojima, K. Ogawa et al., "A proteomic approach for the diagnosis of "Oketsu" (blood stasis), a pathophysiologic concept of Japanese traditional (Kampo) medicine," Evidence-based Complementary and Alternative Medicine, vol. 5, no. 4, pp. 463-474, 2008.

[7] J. K. Nicholson, J. Connelly, J. C. Lindon, and E. Holmes, "Metabonomics: a platform for studying drug toxicity and gene function," Nature Reviews Drug Discovery, vol. 1, no. 2, pp. 153$161,2002$.

[8] J. K. Nicholson, J. C. Lindon, and E. Holmes, “"Metabonomics”: understanding the metabolic responses of living systems to pathophysiological stimuli via multivariate statistical analysis of biological NMR spectroscopic data," Xenobiotica, vol. 29, no. 11, pp. 1181-1189, 1999.

[9] J. T. Brindle, H. Antti, E. Holmes et al., "Rapid and noninvasive diagnosis of the presence and severity of coronary heart disease using $1 \mathrm{H}-\mathrm{NMR}$-based metabonomics," Nature Medicine, vol. 8, no. 12, pp. 1439-1444, 2002.

[10] S. Sun, J. Dai, W. Wang et al., "Metabonomic evaluation of ZHENG differentiation and treatment by Fuzhenghuayu tablet in hepatitis-B-caused cirrhosis," Evidence-Based Complementary and Alternative Medicine, vol. 2012, Article ID 453503, 8 pages, 2012. 
[11] Y. Wang, Z. F. Li, J. X. Chen et al., "Study of mini-pig serum of coronary heart disease (chronic myocardial ischemia) with syndrome of blood stasis based on nuclear magnetic resonance metabolomics," Chinese Journal of Analytical Chemistry, vol. 39, no. 8, pp. 1274-1278, 2011.

[12] B. Yan, A. JiYe, H. P. Hao et al., "Metabonomic phenotype and identification of "heart blood stasis obstruction pattern" and "qi and yin deficiency pattern" of myocardial ischemia rat models," Science in China, Series C: Life Sciences, vol. 52, no. 11, pp. 10811090, 2009.

[13] X. Q. Huang, Q. W. Chen, G. J. Yang et al., "Metabolic profiling study of yang deficiency syndrome in hepatocellular carcinoma by H1 NMR and pattern recognition," Evidence-Based Complementary and Alternative Medicine, vol. 2012, Article ID 843048, 6 pages, 2012.

[14] G. Theodoridis, H. G. Gika, and I. D. Wilson, "LC-MSbased methodology for global metabolite profiling in metabonomics/metabolomics," Trends in Analytical Chemistry, vol. 27, no. 3, pp. 251-260, 2008.

[15] Q. Huang, P. Yin, X. Lu, H. Kong, and G. Xu, "Applications of chromatography-mass spectrometry in metabonomics," Chinese Journal of Chromatography, vol. 27, no. 5, pp. 566-572, 2009.

[16] M. M. Koek, R. H. Jellema, J. van der Greef, A. C. Tas, and T. Hankemeier, "Quantitative metabolomics based on gas chromatography mass spectrometry: status and perspectives," Metabolomics, vol. 7, no. 3, pp. 307-328, 2011.

[17] D. S. Wishart, M. J. Lewis, J. A. Morrissey et al., "The human cerebrospinal fluid metabolome," Journal of Chromatography, vol. 871, no. 2, pp. 164-173, 2008.

[18] "Nomenclature and criteria for diagnosis of ischemic heart disease. Report of the joint international society and federation of cardiology/world health organization task force on standardization of clinical nomenclature," Circulation, vol. 59, no. 3, pp. 607-609, 1979.

[19] Subcommittee of Cardiovascular Diseases of China Society of Integrated Traditional Chinese and Western Medicine, "Criteria for TCM syndrome differentiation of patients with coronary heart disease," Chinese Journal of Integrated Traditional and Western Medicine, vol. 11, no. 5, pp. 257-258, 1991.

[20] J. Trygg, E. Holmes, and T. Lundstedt, "Chemometrics in metabonomics," Journal of Proteome Research, vol. 6, no. 2, pp. 469-479, 2007.

[21] J. A. Westerhuis, T. Kourti, and J. F. Macgregor, "Analysis of multiblock and hierarchical PCA and PLS models," Journal of Chemometrics, vol. 12, no. 5, pp. 301-321, 1998.

[22] M. Sun, X. Q. Gao, D. W. Zhang et al., "Identification of biomarkers for unstable angina by plasma metabolomic profiling," Molecular BioSystems, vol. 9, no. 12, pp. 3059-3067, 2013.

[23] X. Wei, L. Wan, and S. M. Liu, "Plasma metabolomics study on blood stasis syndrome of coronary heart disease," Journal of Traditional Chinese Medicine, vol. 54, pp. 587-591, 2013.

[24] M. White, "Mediators of inflammation and the inflammatory process," Journal of Allergy and Clinical Immunology, vol. 103, no. 3, pp. S378-S381, 1999.

[25] B. Samuelsson, "Leukotrienes: mediators of immediate hypersensitivity reactions and inflammation," Science, vol. 220, no. 4597, pp. 568-575, 1983.

[26] J. A. Burke, R. Levi, Z. G. Guo, and E. J. Corey, "Leukotrienes C4, D4 and E4: effects on human and guinea-pig cardiac preparations in vitro," Journal of Pharmacology and Experimental Therapeutics, vol. 221, no. 1, pp. 235-241, 1982.
[27] J. R. Moffett and M. A. Namboodiri, "Tryptophan and the immune response," Immunology \& Cell Biology, vol. 81, no. 4, pp. 247-265, 2003.

[28] S. J. Szabo, B. M. Sullivan, S. L. Peng, and L. H. Glimcher, "Molecular mechanisms regulating Th1 immune responses," Annual Review of Immunology, vol. 21, pp. 713-758, 2003.

[29] M. C. Cuffy, A. M. Silverio, L. F. Qin et al., "Induction of indoleamine 2,3-dioxygenase in vascular smooth muscle cells by interferon- $\gamma$ contributes to medial immunoprivilege," The Journal of Immunology, vol. 179, no. 8, pp. 5246-5254, 2007.

[30] K. Schröcksnadel, B. Wirleitner, C. Winkler, and D. Fuchs, "Monitoring tryptophan metabolism in chronic immune activation," Clinica Chimica Acta, vol. 364, no. 1-2, pp. 82-90, 2006.

[31] P. Jiang, W. Dai, S. Yan et al., "Potential biomarkers in the urine of myocardial infarction rats: a metabolomic method and its application," Molecular BioSystems, vol. 7, no. 3, pp. 824-831, 2011.

[32] G. J. Wang, A. Jiye, and B. Yan, "Metabonomic investigation on Traditional Chinese Medicine patterns of coronary artery disease," World Science and Technology/Modernization of Traditional Chinese Medicine and Materia Medica, vol. 11, no. 1, pp. 127-133, 2009.

[33] K. Pellegrin, G. Neurauter, B. Wirleitner, A. W. Fleming, V. M. Peterson, and D. Fuchs, "Enhanced enzymatic degradation of tryptophan by indoleamine 2,3-dioxygenase contributes to the tryptophan-deficient state seen after major trauma," Shock, vol. 23, no. 3, pp. 209-215, 2005.

[34] K. Schroecksnadel, S. Kaser, M. Ledochowski et al., "Increased degradation of tryptophan in blood of patients with rheumatoid arthritis," The Journal of Rheumatology, vol. 30, no. 9, pp. 19351939, 2003.

[35] B. Widner, F. Leblhuber, and D. Fuchs, "Increased neopterin production and tryptophan degradation in advanced Parkinson's disease," Journal of Neural Transmission, vol. 109, no. 2, pp. 181-189, 2002.

[36] Y. Wang, S. Guo, C. Li et al., "Analysis of plasma metabonomics of mini-swines with qi deficiency and blood stasis syndrome due to chronic myocardial ischemia," Journal of Chinese Integrative Medicine, vol. 9, no. 2, pp. 158-164, 2011.

[37] Y. P. Qi, H. W. Gu, Y. L. Song et al., "Metabolomics study of resina draconis on myocardial ischemia rats using ultraperformance liquid chromatography/quadrupole time-of-flight mass spectrometry combined with pattern recognition methods and metabolic pathway analysis," Evidence-Based Complementary and Alternative Medicine, vol. 2013, Article ID 438680, 10 pages, 2013.

[38] C. Murr, T. B. Grammer, and A. Meinitzer, "Immune activation and inflammation in patients with cardiovascular disease are associated with higher phenylalanine to tyrosine ratios: the ludwigshafen risk and cardiovascular health study," Journal of Amino Acids, vol. 2014, Article ID 783730, 6 pages, 2014.

[39] B. Tavazzi, A. P. Batocchi, A. M. Amorini et al., "Serum metabolic profile in multiple sclerosis patients," Multiple Sclerosis International, vol. 2011, Article ID 167156, 8 pages, 2011.

[40] H. Yaoita, A. J. Fischman, H. W. Strauss, T. Saito, E. Sato, and Y. Maruyama, "Uridine: a marker of myocardial viability after coronary occlusion and reperfusion," The International Journal of Cardiac Imaging, vol. 9, no. 4, pp. 273-280, 1993.

[41] L. Xiang, P. Jiang, C. Zhan et al., "The serum metabolomic study of intervention effects of the traditional Chinese medicine Shexiang Baoxin Pill and a multi-component medicine polypill 
in the treatment of myocardial infarction in rats," Molecular BioSystems, vol. 8, no. 9, pp. 2434-2442, 2012.

[42] F. J. Dowell, C. A. Hamilton, J. McMurray, and J. L. Reid, "Effects of a xanthine oxidase/hypoxanthine free radical and reactive oxygen species generating system on endothelial function in New Zealand white rabbit aortic rings," Journal of Cardiovascular Pharmacology, vol. 22, no. 6, pp. 792-797, 1993.

[43] H. S. Zhang, Y. H. Jia, and H. Y. Hua, "Study on phlegm syndrome and blood stasis syndrome in coronary artery disease with angina pector is based on metabolomics of urine," Chinese Journal of Basic Medicine in Traditional Chinese Medicine, vol. 161, no. 2, pp. 126-128, 2010.

[44] L. K. Niskanen, D. E. Laaksonen, K. Nyyssönen et al., "Uric acid level as a risk factor for cardiovascular and all-cause mortality in middle-aged men: a prospective cohort study," Archives of Internal Medicine, vol. 164, no. 14, pp. 1546-1551, 2004.

[45] P. E. Puddu, M. Lanti, A. Menotti et al., "Serum uric acid for short-term prediction of cardiovascular disease incidence in the Gubbio population study," Acta Cardiologica, vol. 56, no. 4, pp. 243-251, 2001.

[46] V. G. Athyros, M. Elisaf, A. A. Papageorgiou et al., "Effect of statins versus untreated dyslipidemia on serum uric acid levels in patients with coronary heart disease: a subgroup analysis of the greek atorvastatin and coronary-heart-disease evaluation (GREACE) study," American Journal of Kidney Diseases, vol. 43, no. 4, pp. 589-599, 2004.

[47] S. Burakowski, R. T. Smoleński, J. Bellwon, A. Kubasik, D. Ciećwierz, and A. Rynkiewicz, "Exercise stress test and comparison of ST change with cardiac nucleotide catabolite production in patients with coronary artery disease," Cardiology Journal, vol. 14, no. 6, pp. 573-579, 2007.

[48] G. K. Glantzounis, E. C. Tsimoyiannis, A. M. Kappas, and D. A. Galaris, "Uric acid and oxidative stress," Current Pharmaceutical Design, vol. 11, no. 32, pp. 4145-4151, 2005.

[49] W. H. Liu, Q. Zhang, X. Z. Yan et al., "Metabonomics study on phlegm and blood stasis evolution of hyperlipidemia and atherosclerosis," Journal of Traditional Chinese Medicine, vol. 49, no. 8, pp. 738-741, 2008. 


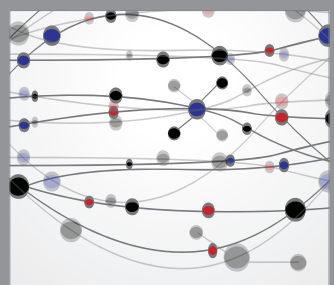

The Scientific World Journal
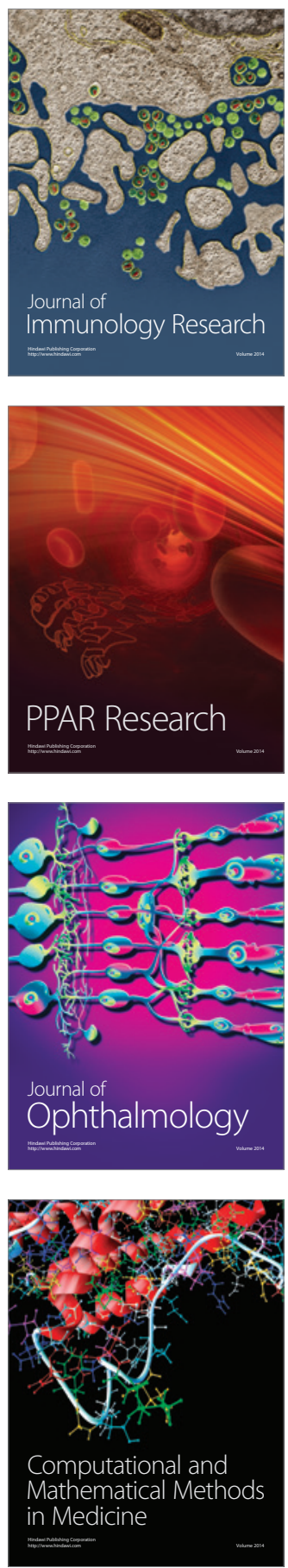

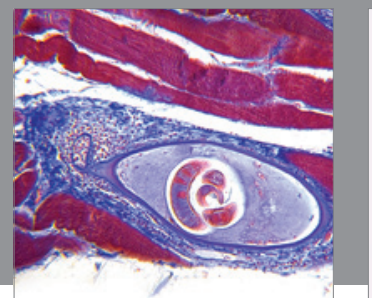

Gastroenterology

Research and Practice
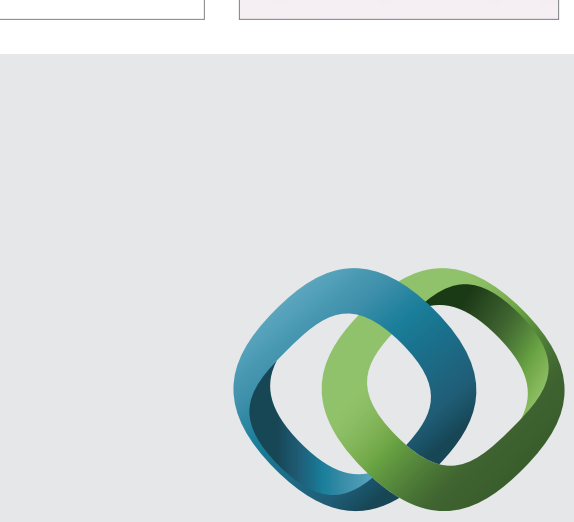

\section{Hindawi}

Submit your manuscripts at

http://www.hindawi.com
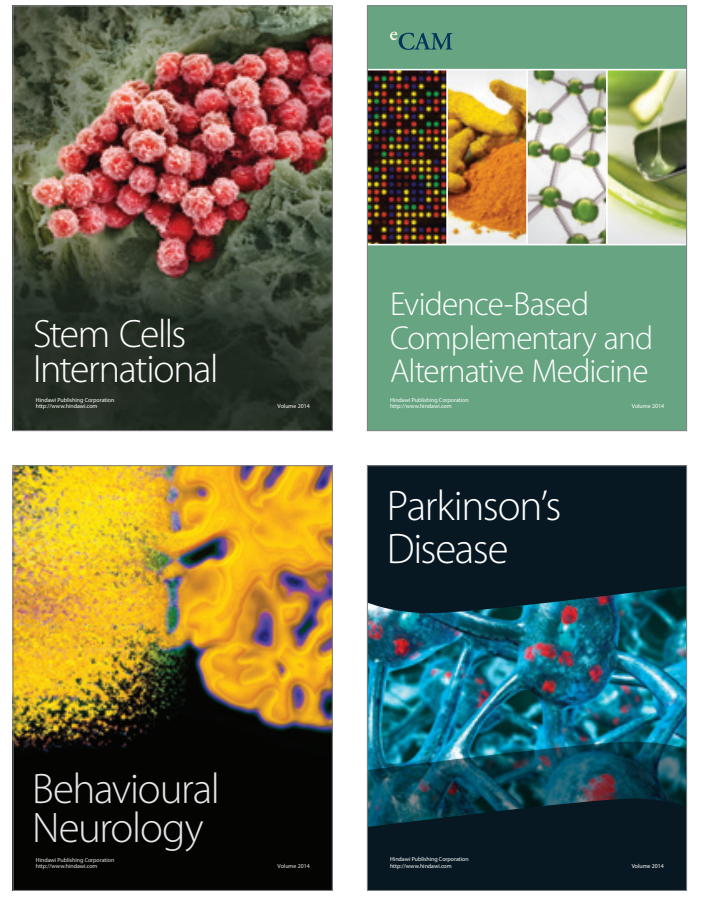
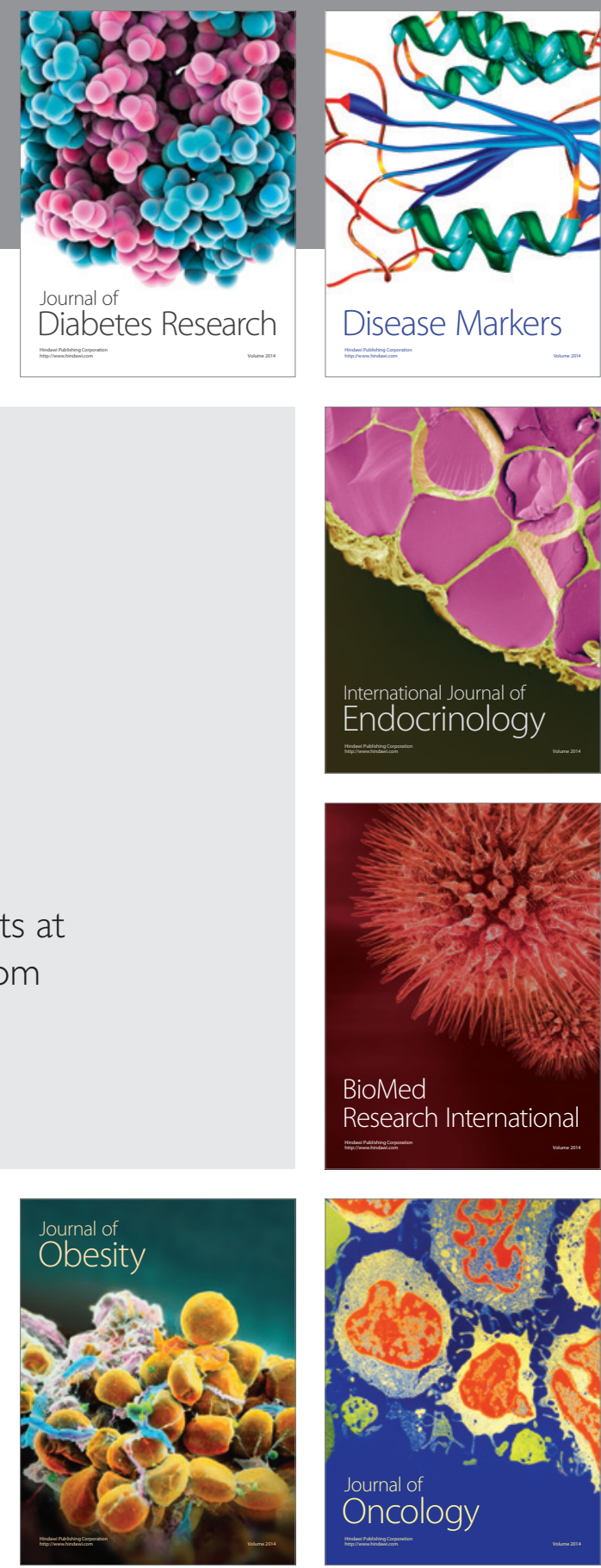

Disease Markers
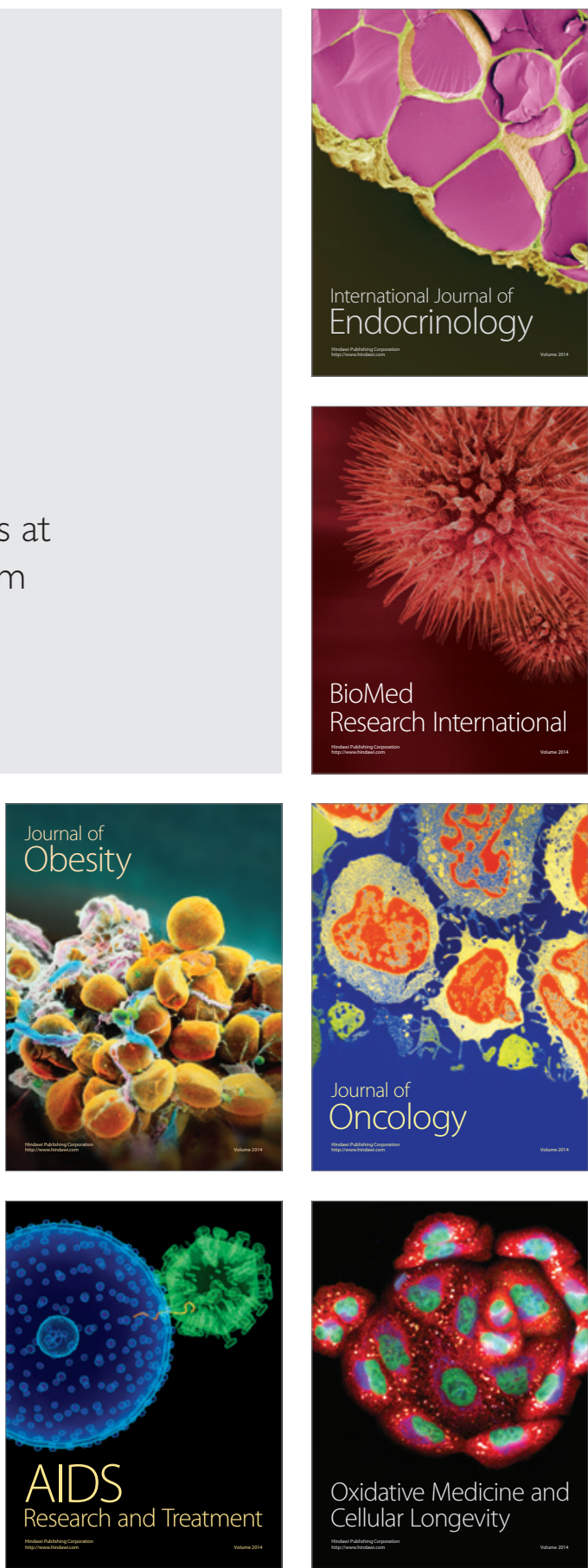